\title{
Bibliografía \\ de Antonio José Cavanilles (1745-1804) y de los estudios sobre su vida y su obra
}

\author{
José M. LÓPEZ PIÑERO \\ MARÍA Luz LÓPEZ TERRADA
}

\section{IMPRESOS}

Libros y folletos

1. Observations... sur l'article Espagne de la nouvelle Encyclopédie.

París, Chez Alex Jombert jeune. Imp. de Didot l'ainé, 1784.

$8 .^{\circ}, 2+155 \mathrm{p}$

2. Observaciones sobre el artículo España de la Nueva Encyclopedia. Traducidas al castellano por don Mariano Ribera.

Madrid, Imprenta Real, 1784.

$8 .^{\circ}, 115 \mathrm{p}$.

3. Dissertatio botanica de Sida, et de quibusdam plantis quae cum illa affinitatem habent.

Parisiis, apud Franciscum Amb. Didot, 1785.

4. ${ }^{\circ}$, 1 - 47 p.; lám. I -XIII.

4. Über der gegenwartigen Zustand von Spanien. Aus der französischen Urschrift des spanischen Verfassers.

Berlín, bei J. F. Unger, 1785.

$8 .^{\circ}, 16+158 \mathrm{p}$.

5. Mémoires sur la culture de certaines Malvacées \& I'usage économique qu'on pourra retirer de leurs fibres.

[París, s.i.] 1786.

4. ${ }^{\circ}, 10 \mathrm{p}$.

6. Secunda dissertatio botanica, De Malva, Serra, Malope, Lavatera, Alcea, Althea et Malachra. Accedunt Sidae mantissa et tentamina de Malvarum atque Abutilonis fibris in usus oeconomicos praeparandis.

Parissis, apud Franciscum Amb. Didot, 1786.

4., 43 - 106 p. + 6 p.; lám. XIV - XXXV + lám. A. 


\section{JOSE M. LOPEZ PIÑERO Y MARIA LUZ LOPEZ TERRADA}

7. Tertia dissertatio botanica, De Ruizia, Assonia, Dombeya, Pentapete, Malvavisco, Pavonia, Hibisco, Laguna, Cienfuegosia, Quararibea, Pachira, Hugonia, et Monsonia. Parissis, Apud Franciscum Amb. Didot, 1787.

4. ${ }^{\circ}, 107$ - 186 p.; lám. XXXVI - LXXIV.

8. Quarta dissertatio botanica, De Geranio, CXXVIII species complectens, XLIX tabulis incisas.

Parissis, apud Franciscum Amb. Didot, 1787.

4. ${ }^{\circ}, 189$ - 266 p.; lám. LXXV - CXXIV.

9. Quinta dissertatio botanica, De Sterculia, Kleinhovia, Ayenia, Buttneria, Bombace, Adansonia, Crinodendro, Aytonia, Malachodendro, Stewartia et Napaea. Accedit praecedentium dissertatione mantissa, XXXVI tabulis aere incisis ornata.

Parissis, Apud Franciscum Amb. Didot, 1788.

4. ${ }^{\circ}, 267$ - 303 p.; lám. CXXV - CLIX.

10. Sexta dissertatio botanica, De Camellia, Gordonia, Morisona, Gossypio, Waltheria, Melochia, Mahernia, Hermannia, Urena, Halesia, Styrace, Galxia, Ferraria, et Sisyrinchio. Accedit mantisa tertia. XLI tabulis aere incisis ornata.

Parissis, Franciscum Amb. Didot, 1788.

4. ${ }^{\circ}, 304$ - 357 p.; lám. CLX - CC.

11. Carta... en respuesta a la que se insertó en la segunda parte del Memorial Literario del mes de Septiembre de 1788, donde se hace crítica de sus Disertaciones botánicas por uno que se titula vecino de Lima.

Madrid, por la Viuda de Ibarra, 1789

$8 .^{\circ}, 16 \mathrm{p}$.

12. Septima dissertatio botanica, quatordecim genera monadelphia continens, XXIV tabulis accurate delineata.

Parissis, Franciscum Amb. Didot, 1789.

$4 .^{\circ}, 355$ - 378; lám. CCI - CCXXIV.

Contiene además (p. 379 -396): Observationes in quintum fasciculum D. L'Héritier.

13. Observationes in quintum fasciculum $D$. L'Héritier.

[París, s.i., 1789].

$4 .^{\circ}, 18 \mathrm{p}$.

Separata de Septima Dissertatio botanica, quatordecim genera monadelphia continens... Parissis, Franciscum Amb. Didot, 1789, p. 379-396.

14. Observations... sur le cinquième fascicule de $M$. L'Héritier.

[París, s.i., 1789].

$4 .^{\circ}, 10 \mathrm{p}$.

Separata, con nueva paginación, de Observations sur la physique, sur l'histoire naturelle et sur les arts, 34 (1789), 183-192.

15. Octava dissertatio botanica, Erythroxylon et Malpighia complectens, XVIII tabulis omata. Parissis, Franciscum Amb. Didot, 1789.

4. ${ }^{\circ}, 397$ - 414 p.; lám. CCXXV - CCXLII.

16. Nona dissertatio botanica, De Banisteria, Triopteride, Molina et Flabellaria, XXII tabulis ornata. Superiorum permissu.

Matriti, Ex Typographia Regia, 1790.

4. ${ }^{\circ}, 415$ - 436 p.; lám. CCXLIII - CCLXIV.

17. Decima dissertatio botanica, De Passiflora, XXXII tabulis ornata... Superiorum permissu.

Matriti, Ex Typographia Regia, 1790.

4. ${ }^{\circ}, 437$ - 463 p.; lám. CCLXV - CCXCVI. 
18. Monadelphiae Classis Dissertationes Decem.

Matriti, Ex Typographia Regia, Postrant: Matriti apud D. Antonium Baylo, Parissis apud D. Firminum Didot, 1790.

3 vols.

Vol. I: $8 .^{\circ}, 1$ h. [en verso, grabado con el retrato de Cavanilles: "Antonius Joseph Cavanilles Hispano-Valentinus. Natus Valentiae die 16. Januarii 1745"] + portada + 1-4 p. [«Praefatio in universum opus $]+5-10$ p. [«Characterum tabula»] + 2 h. ["Generum Monadelphiae tabula analitica»] + 463 p. [texto de $3,6-10,12,15-17$, con paginación correlativa y portadas propias, igual que en las ediciones individuales de las Dissertationes] +1 p. [ «Index generum totius operis"].

Vol II: Fol. lám A + lám I-ĊLIX.

Vol III: Fol. lám CLX- CCXVI.

19. Icones et descriptiones plantarum quae aut sponte in Hispania crescunt, aut in hortis hospitantur.

Matriti, ex Typographia Regia, 1791-1801.

Fol. 6 vols.

Vol. I (1791): 4+68+4 p.; láms. 1- 100.

Vol. II (1793): 4+79+5 p.; láms. 101- 200.

Vol. III (1794): $10+52+2+4$ p.; láms. $201-300$.

Vol. IV (1797): 4+82 p.; láms. 301- 400.

Vol. V (1799): 4+74 p.; láms. 401- 500.

Vol. VI (1801): 4+98 p. láms. 501- 600.

20. Observaciones sobre la Historia Natural, geografía, agricultura población y frutos del Reyno de Valencia.

Madrid, en la Imprenta Real, 1795-1797.

Fol. 2 vols.

Vol. I (1795): 16+236 p.; 28 láms.

Vol. II (1797): 4+335 p.; 25 láms.

21. Colección de papeles sobre controversias botánicas de... con algunas notas del mismo a los escritos de sus antagonistas.

Madrid, Imprenta Real, 1796.

$8 .^{\circ}, 274 \mathrm{p}$

22. Suplemento a las observaciones sobre el cultivo del arroz en el Reyno de Valencia y su influencia en la salud pública, en respuesta a la contestación de D. Vicente Ignacio Franco.

Madrid, Imprenta Real, 1798.

$8 .^{\circ}, 38 \mathrm{p}$.

23. Descripción de las plantas que... demostró en las lecciones públicas del año 1801 y 1802, precedida de los principios elementales de la botánica.

Madrid, Imprenta Real, 1802.

$8^{\circ}, 136+625$ p. + l h.

Según Colmeiro (170, p.103) y Palau Dulcet (207, n. ${ }^{\circ}$ 50576), hay otra edición en Madrid, Imprenta Real, 1803, con un título algo diferente.

Según HERNÁNDEZ MOREJÓN (184, vol.VII, p.397), existe una traducción francesa.

24. Discurso que... leyó en el Real Jardín Botánico de esta Corte el 13 de abril de 1803. [Madrid, s.i., 1803].

4. ${ }^{\circ}, 20 \mathrm{p}$.

25. Elenchus plantarum Horti Regii Botanici Matritensis, anno MDCCCIII.

[Madrid, s.i., 1803].

$16 .^{\circ}, 40 \mathrm{p}$.

Asclepio-Vol. XLVII-1-1995

243 


\section{JOSE M. LOPEZ PIÑERO Y MARIA LUZ LOPEZ TERRADA}

26. Principi elementari di Botanica... tradotti allo spagnuolo dal dott. Dom. Viviani... Genova, Stamperia della Società Médica di Emulazione, 1803.

$4 .^{\circ}, 144 \mathrm{p}$.

27. Principi elementari di botanica... tradotti dallo spagnuolo dal dott. DomenicoViviani... Col'aggiunta di un nuovo saggio sopra i sistemi di Tournefort, Linneo, e Jussieu et altri nuove annotazioni...

Genova, Stamperia Frugoni, 1808.

4. ${ }^{\circ}, 199 \mathrm{p}$.

Según Colmeiro (170, p. 103) y Palau Dulcet (207, n. ${ }^{\circ}$ 50576), la fecha de impresión es 1804.

28. Descripción de las plantas que... demostró en las lecciones públicas del año 1801 y 1802, precedida de los elementos de botánica.

Madrid, Imprenta Real, 1827.

$4 .^{\circ}, 136+625 \mathrm{p}$.

29. Observaciones sobre la Historia Natural, Geografía, Agricultura, Población y frutos del Reyno de Valencia. Segunda edición al cuidado de José Manuel Casas Torres. Zaragoza, C.S.I.C., 1958.

$4 .^{\circ}, 2$ vols.

30. Icones et descriptiones plantarum, quae aut sponte in Hispania crescunt, aut in hortis hospitantur. Facsimile edition. Introductory note by F.A. Stafleu.

Lehre, J. Cramer, 1965. [Historiae naturalis clasica, vol. 42].

Fol. 6 vols.

31. Excavaciones en Calpe (1797).

Santiago de Compostela, Seminario de Arqueología, 1970. [Studia Archeologica, vol. 5; Materiales para la Historia de la Arqueología Española, vol. 1].

$4 .^{\circ}, 36 \mathrm{p}$.

32. Observaciones sobre la historia natural, geografía, agricultura, población y frutos del Reyno de Valencia.

Valencia, Albatros, 1972

Fol. 2 vols.

33. Observaciones sobre la historia natural, geografía, agricultura, población y frutos del Reyno de Valencia.

Valencia, Albatros, 1975

Fol. 2 vols.

34. Plantas Valencianas... Justificación por Josep Palacios.

Valencia, Cosmos, 1977.

Fol. 3 h. + 12 p.; 71 lám.

Reproducción facsímil de' fragmentos de 19 y 20.

35. Observaciones sobre la historia natural, geografía, agricultura, población y frutos del Reyno de Valencia.

Valencia, Albatros, 1978

Fol. 2 vols.

36. Observaciones sobre la historia natural, geografía, agricultura, población y frutos del Reyno de Valencia.

Valencia, Albatros, 1979.

Fol. 2 vols.

37. Observaciones sobre la historia natural, geografía, agricultura, población y frutos del Reyno de Valencia.

Valencia, Albatros, 1981.

Fol. 2 vols. 
38. Cartas a José Viera y Clavijo. Introduccion y notas de Alejandro Cioranescu. Santa Cruz de Tenerife, Cabildo insular de Tenerife, 1981. $4 .^{\circ}, 158 \mathrm{p}$.

39. Antoni Josep Cavanilles. Calendari any 1983.

València, Acció Cultural del País Valencià, 1982.

Fol. 2 h.; 6 láms.

Reproducción facsímil de seis grabados de 19.

40. Observaciones sobre la historia natural, geografía, agricultura, población y frutos del Reyno de Valencia.

Valencia, Albatros, 1983.

Fol. 2 vols.

41. 10 estampas de A.J. Cavanilles.

Madrid, Real Jardín Botánico, 1983.

Fol. 10 láms.

Reproducción facsímil de cinco grabados de $\mathbf{2 0}$, de tres de $\mathbf{1 8}$ y de dos de 19 .

42. Observaciones sobre la historia natural, geografía, agricultura, población y frutos del Reyno de Valencia.

Valencia, Albatros, 1984.

Fol. 2 vols.

43. Observaciones sobre la historia natural, geografía, agricultura, población y frutos del Reyno de Valencia.

Valencia, Albatros, 1985.

Fol. 2 vols.

44. Las comarcas de los Serranos y el Rincón de Ademuz según Cavanilles y Madoz. Valencia, Mancomunidad del Alto Turia, 1987.

Fol. 140 p.

45. Observaciones sobre la historia natural, geografía, agricultura, población y frutos del Reyno de Valencia.

Valencia, Albatros, 1987.

Fol. 2 vols.

46. Observaciones sobre la historia natural, geografía, agricultura, población y frutos del Reyno de Valencia.

Valencia, Albatros, 1989.

Fol. 2 vols.

47. Hortus Regius Matritensis

En: J. CARRETE PARRONDO, Difusión de la ciencia en la España ilustrada estampas de la Real Calcografía. Madrid, C.S.I.C., 1989, p. 70-116.

Edición de 45 grabados, correspondientes a 137.

48. Hortus Regius Matritensis o Descripción de algunas plantas nuevas o raras cultivadas en el Real Jardín Botánico de Madrid o conservadas en su herbario. Con una introducción histórica por Francisco Pelayo y Ricardo Garilleti, una explicación de los métodos de reproducción calcográfica además de notas sobre la biografía de los hermanos valencianos López Enguídanos por Juan Carrete Parrondo y la transcripción de los textos latinos de Cavanilles por Francisco Javier Fernández Casas y Francisco Javier Sánchez García.

Madrid, edición a cargo de Cartonajes Suñer, S.A. y del Real Jardín Botánico (C.S.I.C.), 1991

Fol. 2 h. +43 p. +50 p. +1 h. +100 láms. +1 h.

Edición de 115 y 116.

Asclepio-Vol. XLVII-1-1995 
49. Observaciones sobre la historia natural, geografía, agricultura, población y frutos del Reyno de Valencia.

Castellón, Caja de Ahorros y Monte de Piedad de Castellón, 1991.

Fol. 2 vols.

Incluye (vol. 1, p. 1-10), el estudio introductorio de J. Mateu Bellés, «El viaje de Cavanilles por el Reyno de Valencia (1791-1793)».

50. Observaciones sobre la historia natural, geografía, agricultura, población y frutos del Reyno de Valencia.

Valencia, Albatros, 1993.

Fol. 2 vols.

51. Icones et descriptiones plantarum quae aut sponte in Hispania crescunt, aut in Hortis hospitantur.

Valencia, Consellería de Cultura, 1995.

Fol. 6 vols.

Incluye (vol. I, p. IX-XLVIII), un estudio introductorio de M. Costa Taléns, en valenciano y castellano.

52. Observaciones sobre la historia natural, geografía, agricultura, población y frutos del Reyno de Valencia.

Valencia, Albatros, 1995.

Fol. 2 vols.

\section{Artículos de Revista}

53. Observations... sur le cinquième fascicule de M.L. Héritier, Observations sur la physique, sur l'histoire naturelle et sur les arts, 34 (1789), 183-192.

54. Observationes in quintum fasciculum D. L'Héritier, Magazin für die Botanik, 3 (1790), 42-60.

55. Observaciones sobre el cultivo del arroz en el Reino de Valencia, Memorias de la Real Academia Médica de Madrid, 1 (1797), 99-128.

56. Descripción de cinco géneros nuevos y de otras plantas, Anales de Historia Natural, 1 (1799), 33-45 +5 lám.

57. De los géneros Goodenia y Scaerola, Anales de Historia Natural, 1 (1799), 89-107.

58. Nuevos caracteres genéricos de los helechos por Smith, Anales de Historia Natural, 1 (1799), 108-115.

59. Historia Natural de las palomas de España y especialmente de Valencia, Anales de Historia Natural, 1 (1799), 146-176.

60. [Del terremoto que se observó en el Reyno de Quito en 1797], Journal de physique, de chimie, d'histoire naturelle et des arts, 49 (1799), 230-232.

61. Cultivo del arroz, Semanario de Agricultura, 6 (1799), 245-252.

Resumen de $\mathbf{5 5}$.

62. Cultivo del naranjo [en la huerta de Orihuela], Semanario de Agricultura, 5 (1799), 51-56.

Resumen de un fragmento de $\mathbf{2 0}$.

63. Observaciones sobre el suelo, naturales y plantas del Puerto Jackson y Bahía Botánica, Anales de Historia Natural, 1 (1800), 181-239.

64. Observaciones botánicas, Anales de Historia Natural, 1 (1800), 240-243.

65. Materiales para la historia de la Botánica, Anales de Historia Natural, 2 (1800), 3-57.

66. Del terremoto que se observó en el Reyno de Quito en 1797, Anales de Historia Natural, 2 (1800), 91-104.

67. Descripción de algunas plantas nuevas, Anales de Historia Natural, 2 (1800), 104-124 
68. Observaciones sobre algunos vegetales que producen resina elástica, Anales de Historia Natural, 2 (1800), 124-128.

69. Descripción del género Bonplandia y de otras plantas, Anales de Historia Natural, 2 (1800), 131-142.

70. Descripción del género Buena, Anales de Historia Natural, 2 (1800), 171-178.

71. Polvos contra la rabia, Anales de Historia Natural, 2 (1800), 178-195.

72. Del sinfito pétreo, Anales de Historia Natural, 2 (1800), 271-278.

73. Remedio contra la rabia, Semanario de Agricultura, 8 (1800), 250-256. Resumen de $\mathbf{7 1}$.

74. Del cultivo de las chufas, Semanario de Agricultura, 7 (1800), 273-275. Resumen de 82.

75. Sobre el cultivo de algunas especies de malvas y uso económico que se puede hacer de sus fibras, Semanario de Agricultura, 7 (1800), 305-309.

Resumen de una memoria leída en la Académie de Sciences, de París, el 1 de febrero de 1786, y publicada ese mismo año en París (5).

76. De las plantas que el ciudadano Augusto Broussonet colectó en las costas septentrionales de la África y en las Islas Canarias, Anales de Ciencias Naturales, 3 (1801), 5-78.

77. Enfermedad y muerte de un rabioso, Anales de Ciencias Naturales, 3 (1801), 115-126.

78. Enfermedad y muerte de otro rabioso, Anales de Ciencias Naturales, 3 (1801), 126-129.

79. Descripción de los géneros Aeginetia, Rizoa y Castelia, Anales de Ciencias Naturales, 3 (1801), 129-135.

80. Disertación sobre el género Aro por el ciudadano E. P. Ventenat, Anales de Ciencias Naturales, 3 (1801), 136-148.

81. Descripción de dos géneros nuevos de plantas, Anales de Ciencias Naturales, 3 (1801), 230-234.

82. Del cultivo de las chufas, Anales de Ciencias Naturales, 3 (1801), 234-236.

83. Extracto de una memoria sobre la enfermedad y curación de tres hombres mordidos por un perro rabioso, Anales de Ciencias Naturales, 3 (1801), 237-250.

84. Enfermedad y muerte de un hombre que murió rabioso en 1 de Febrero de 1801, Anales de Ciencias Naturales, 3 (1801), 250-260.

85. Funestos efectos de la rabia, Anales de Ciencias Naturales, 4 (1801), 3-11.

86. Fascículo II. De las plantas que el ciudadano A. Broussonet colectó en las costas septentrionales de la África y en las Islas Canarias, Anales de Ciencias Naturales, 4 (1801), 52-109.

87. Suplemento al género Buena, Anales de Ciencias Naturales, 4 (1801), 109-120.

88. Nuevos experimentos que confirman la virtud profiláctica de los polvos vegetales, Anales de Ciencias Naturales, 4 (1801), 225-230.

89. Caso extraordinario en la enfermedad de la rabia, Anales de Ciencias Naturales, 4 (1801), 230-236.

90. Del género nuevo Francoa, Anales de Ciencias Naturales, 4 (1801), 236-239.

91. Extracto del primer fascículo de la obra titulada «Ticinensis horti academici plantae selectae», Anales de Ciencias Naturales, 4 (1801), 239-243.

92. Del género Ugena, Anales de Ciencias Naturales, 4 (1801); 249-256.

93. Suplemento a los artículos sobre la rabia y sus remedios profilácticos, Anales de Ciencias Naturales, 4 (1801), 344-346.

94. Extracto de los cuatro primeros fascículos del Jardín de J. M. Cels, Anales de Ciencias Naturales, 4 (1801), 346-352.

95. De los géneros Cephalophora y Tricycla, Anales de Ciencias Naturales, 5 (1802), 60-64.

96. Discurso que... leyó en el Real Jardín Botánico de esta Corte el $1^{\circ}$ de abril de 1802, Anales de Ciencias Naturales, 5 (1802), 111-134. 


\section{JOSE M. LOPEZ PIÑERO Y MARIA LUZ LOPEZ TERRADA}

97. Medicina Militar. Fragmentos de Colección de Observaciones que R. Desgenettes hizo en el Exército de Oriente, Anales de Ciencias Naturales, 5 (1802), 218-223.

98. Continuación de la obra titulada "Plantarum rariorum Hungariae decades», Anales de Ciencias Naturales, 5 (1802), 223-234.

99. De la cigüeña blanca, Anales de Ciencias Naturales, 5 (1802), 234-44.

100. Continuación de las plantas cultivadas en el Jardín de J. M. Cels, Anales de Ciencias Naturales, 5 (1802), 344-371.

101. Observaciones botánicas, Anales de Ciencias Naturales, 5 (1802), 245-263.

102. Principios de botánica, Semanario de Agricultura, 12 (1802), 209-224, 226-239, 242-256, 261-272, 279-287.

El nombre de Cavanilles no figura en el texto, aunque sí en el índice del volumen de la revista. Félix Muñoz Garmendía, a quien agradecemos la noticia, ha comprobado que consiste en un resumen de la primera parte de 23 .

103. Discurso que... leyó en el Real Jardín Botánico de esta Corte en 13 de Abril de 1803, Anales de Ciencias Naturales, 6 (1803), 119-138.

104. Observaciones botánicas y descripción de algunas plantas nuevas, Anales de Ciencias Naturales, 6 (1803), 323-340.

105. Continuación de las plantas cultivadas en el Jardín de J. M. Cels y extracto de los dos últimos quadernos, Anales de Ciencias Naturales, 6 (1803), 367-370.

106. Del macrocnemo y de algunas plantas descubiertas por los españoles, Anales de Historia Natural, 7 (1804), 55-57.

107. Jardín de la Malmaison, Anales de Ciencias Naturales, 7 (1804), 70-74.

108. Las Liliáceas de P. S. Redouté, Anales de Ciencias Naturales, 7 (1804), 74-80.

109. Discurso sobre algunos Botánicos Españoles del siglo XVI, leído en el Real Jardín Botánico al principiar el curso de 1804, Anales de Historia Natural, 7 (1804), 99-140.

110. Del género nuevo Viviana, Anales de Ciencias Naturales, 7 (1804), 211-212.

111. Adición al artículo del Licenciado Robles, citado en la nota segunda de la pag. 120, Anales de Historia Natural, 7 (1804), 216.

112. Botanical Observations by the late Don Antonio Joseph Cavanilles, translated from the Spanish, Annals of Botany, 1/2 (1805), 409-422.

\section{MANUSCRITOS}

\section{Real Jardín Botánico, Madrid}

113. [Apuntes bibliográficos e históricos].

Legajo de $10 \mathrm{~h}$.

5. ${ }^{a}$ Div. $n .^{\circ} 23(1-10)$

Contiene:

- Notas sobre Pedro Pomar, Pedro Jaime Esteve, Miguel Jerónimo Ledesma, Pedro Ximeno, Luis Collado, Juan Plaza y otros médicos y botánicos valencianos del siglo XVI, procedentes de su lectura de los libros de GASPAR EscolaNO, Décadas de la Historia de la Insigne y Coronada Ciudad y Reyno de Valencia, 10 vols., Valencia, 1610-1611, y de VICENTE XIMENO, Escritores del Reyno de Valencia, 2 vols., Valencia, 1747-1749.

- "Apuntaciones" procedentes de su lectura de las obras de LORENZo PÉREZ, Libro de la Theriaca, Toledo, 1575, y De medicamentorum simplicium et compositorum hodierno aevo apud nostros pharmacopolas extantium delectu, repositione et aetate..., Toledo, 1590. 
- Notas sobre la estancia de Pehr Loffing en América.

- «Apuntaciones» procedentes de su lectura de la obra de BERNABÉ COBO, Historia del Nuevo Mundo, Ms. 1653.

114. [Apuntes varios]

Legajo de $81 \mathrm{~h}$.

5. ${ }^{\mathrm{D}}$ Div. n. ${ }^{\circ} 24$ (1-72).

Contiene:

- Copia manuscrita de un fragmento de las Observaciones sobre la Historia Natural, Geografía, Agricultura, población y frutos del Reyno de Valencia (1795-1797), relativo al Vizcondado de Chelva, Titaguas y Aras.

- Notas sueltas de tema vario: planes de trabajo, listas, textos morales, etc.

- Notas procedentes de la lectura de la continuación de la obra Plantarum rariorum Hungariae decades, que Cavanilles comentó en Anales de Ciencias Naturales.

- Notas sobre crucíferas procedentes de la lectura de la obra de JEAN BAPTISTE DE MONET DE LAMARCK, Flore française, 3 vols., París, 1779, y de CARL PETER THUNBerg, Voyages... au Japon, par le Cap de Bonne-Espirance, les Isles de la Sonde, 2 vols., París, an IV (1796).

- Descripciones de algunas plantas para su curso de 1803. Ocupan las hojas 27 a 64 y siguen el mismo plan que las publicadas, correspondientes a los años 1801 y 1802; en las hojas 60 a 64 hay dibujos a lápiz de Banksia serrata (o conchífera), Banksia gibbosa y Banksia pyriformis, según JOSEPH GAERTNER, De fructibus et seminibus plantarum, 3 vols., Stuttgardiae et Tubingae, 1788-1807.

- Notas procedentes de la lectura de la serie dirigida por ERNST GOTTFRIED BALDINGER, Sylloge selectiorum opusculorum argumenti medico-practici, 6 vols., Goettingae, 1776-1782

- Notas sueltas sobre plantas.

115. [Cien láminas. Hortus Botanicus Matritensis]. Vol. de 100 h.+ 78 láms. sueltas. 5. ${ }^{a}$ Div. n. ${ }^{\circ} 8(90-190,191-268)$.

Contiene:

-100 hojas encuadernadas, en fol. menor, con dibujos a pluma de plantas, originales de Cavanilles [C], José Guío [G] y Antonio Delgado Meneses [D]:

1. Boerhaavia viscosa [G].

2. Boerhaavia arborescens [G].

3. Ziziphora spicata [G].

4. Papophorum bulbosum. Matriti die 11 martii 1804. ex sicco [C].

5. 1.Papophorum phleoides. 2. cynosurus tenellus. 3. cynosurus gracillis. Matriti die 17 martii 1804. [C].

6. Mustelia arundinacea. Matriti die 20 martii 1804. [C].

7. Boldoa purpurascens [C].

8. Mentha divaricata [G].

9. Linaria elegans $[\mathrm{C}]$ :

10. Vitex pendula [G].

11. Gonatocarpus mucronatus Thub. [D].

12. Nicotiana scabra [G].

13. Ipomaea crassifolia. Matriti in R. h. die 26 Sept. 1801 ex planta ex seminibus lectis in Guayaquil [C].

14. Ipomaea peduncularis. Matriti die 14 Novembris 1802 ex planta viva [C].

15. Ipomaea horrida. [C].

16. Ipomoea stylifera [G].

17. Capsicum microcarpum. Matriti die 4 Octobris 1802. ex planta viva. [C]. 


\section{JOSE M. LOPEZ PIÑERO Y MARIA LUZ LOPEZ TERRADA}

18. Capsicum violaceum. Matriti die 18 Octobris 1802. ex vivo. [C].

19. Macrocnemum tetrandan. [C].

20. Salsola eriophora [G].

21. Atriplex verticillata [G].

22. Eruca hispida [G].

23. Sisymbrium contortum [G]

24. Sisymbrium crassifolium [G]

25. Amaryllis pygmaea [G].

26. Scilla lutea [G].

27. Agave brachystachia [G].

28. Agave spicata [G]

29. Cassia torosa [sin firma]

30. Cassia papilionacea [G].

31. Pomaria glauca [G].

32. Caesalpinia pectinata [G].

33. Viviania marifolia [G].

34. Agrostemma parviflora [G]

35. Balbisia verticillata $[\mathrm{C}]$.

36. Mimosa torquata [G].

37. Mimosa siliquastrum [G]

38. Mimosa leptophylla. [G].

39. Mimosa aculeaticarpa [G].

40. Luziola peruviana. Matriti die 12 Martii 1801. ex sicco. [C].

41. Euphorbia pithyusa [G].-

42. 1. Ranunculus rotundifolius. 2. Ranunculus setaceus [C].

43. Passiflora hibiscifolia [G].

44. Nevrada procumbens [G]

45. Sida deflexa. [G].

46. Anoda acerifolia [G]

47. Malva triloba [G].

48. Lavatera acerifolia [G]

49. Malachra livida [G]

50. Hibiscus hispidulus $[\mathrm{G}]$

51. Clementea nitida $[\mathrm{C}]$.

52. Clitoria speciosa. Matriti in horto R. die 28 Sept. 1801 ex planta ennata ex seminibus misis per D.J. Guio ex Habana. [C].

53. Ornithopus moniliformis [G]

54. Crotalaria pygmaea [G]

55. Ononis compressa [G].

56. Ononis inclusa [G].

57. Athanasia crithmifolia $[\mathrm{G}]$.

58. Tagetes peduncularis. Matriti.in R. h. die 26 Sept. 1801 ex planta viva ennata ex Seminibus e Cumana misis a D. Bompland. [C]

59. Tagetes glandulosa. Die 21 Oct. 1802. ex sicco exemplare lecto in Chile [C].

60. Dyssodia porophylla.[G].

61. Heterosperma ovatifolia $[\mathrm{C}]$.

62. Heterosperma trifida [C].

63. Rudbeckia scabra [G]

64. Melampodium longifolium [G].

65. Lagasca mollis [G].

66. Meniscium serratum [G]. 


\section{BIBLIOGRAFIA DE ANTONIO JOSE CAVANILLES (1745-1804)}

67. Polypodium longifolium [G].

68. Polypodium grammitis [G].

69. Polypodium elegans [G].

70. Polypodium plumarium [G].

71. Tectaria triloba [D]

72. Tectaria falcata [G]

73. Tectaria fraxinea [G].

74. Tectaria calahuala [D].

75. Oleandra neriformis [G]

76. Asplenium fumarioides [D].

77. 1. Asplenium flavelifolium. 2. Asplenium trilobum [D].

78. Diplazium nitidum [G].

79. Diplazium grandiflorum [G].

80. Diplazium juglandiflolium [G].

81. Woodwardia caudata [G].

82. Woodwardia stans [G].

83. Woodwardia radicans [G].

84. Pteris sagittata [G].

85. Pteris cordata [G].

86. Pteris subverticillata [G].

87. Pteris sulphurea [G].

88. Pteris lendigera $[\mathrm{G}]$.

89. 1. Pteris imbricata. 2. Pteris humilis [G].

90. Onoclea glacialis [D].

91. Lindsaea incisa [D].

92. Humata falcata [G].

93. Humata trifoliata [G].

94. Humata ophioglossa [G].

95. Humata pinnatifida [G].

96. Squamaria integra [G].

97. Hymenophyllum cruentum [D].

98. Hymenophyllum pectinatum [D]

99. Trichomanes rhizophylla [D].

100. Angiopteris erecta [G].

- 78 estampas sueltas. Son estampas calcográficas grabadas por un grupo de artistas dirigidos por Tomás López Enguídanos, que corresponden a parte de los dibujos anteriores, entre ellos, los siguientes de Cavanilles: 7, 9, 13-15, 17 . $19,35,42,51-52,58-59,61-62$.

116. [Hortus Matritensis. Descripciones originales, que corresponden a los dibujos existentes en el Jardín Botánico de Madrid].

Legajo de $89 \mathrm{~h}$.

5. ${ }^{a}$ Div. $n .^{\circ} 8$ (1-89).

Contiene los textos descriptivos, en latín, de los dibujos de 115, con la excepción de los correspondientes a los números 1, 2, 8, 11, 16, 37, 49, 50, 76, 77, 91-93.

117. [Hortus Regius Matritensis].

Legajo de $98 \mathrm{~h}$.

5. ${ }^{\mathrm{D}}$ Div. $n .^{\circ} 27$ (1-98).

Copia incompleta del anterior.

118. [Lista de las Quinas que hay en el herbario de Cavanilles.] 5. ${ }^{\mathrm{a}}$ Div. $\mathrm{n}^{\circ} 8(274)$ 


\section{JOSE M. LOPEZ PIÑERO Y MARIA LUZ LOPEZ TERRADA}

119. Monadelphiae Classis Dissertationes decem.

Cit. por ÁlVAREZ LÓPEZ (144, p. 57).

Dibujos originales de las Dissertationes, todos ellos de Cavanilles, a excepción de tres correspondientes a la Dissertatio botanica de Sida, debidos a L. Fossier.

\section{Real Academia de Medicina, Madrid}

120. ¿Hay géneros naturales en las plantas? (1794).

Cit. por COLMEIRO $(164,86)$.

\section{Archivo Familiar}

121. [Apuntes Botánicos].

Archivo-Carpeta, 9, 1

Está encabezado por la siguiente nota autógrafa: «Empezé el estudio de la Botánica en el Otoño de 1780 y las descripciones contenidas en este tomo en 1782 que continué en los dos siguientes de 83 y 84 . Hacíalas como aprendiz, sin el conocimiento que luego adquiriría tratando con los Sres. de Jussieu, Thauin, Lamarck, Desfontaines, Beaupres, etc., viendo los Jardínes de Trianon y M. Monnier en Versalles; de Bellevue, Real de París y los de Cels y Saint Germain en la misma capital, otros famosos de Sevres, muchos de Brusellas, y las plantas espontáneas de los sitios por donde iba viajando. Revolví Herbarios y autores; rectifiqué mis ideas y notando yerros y faltas en autores empezé a preparar mis obras, publicando la primera en 1785».

122. [Apuntamiento de Lógica-Crítica]

Archivo-Carpeta 9, 6 .

123. Apuntamientos lógicos o Compendio del Arte lógico-crítico, para el uso del Excelentísimo Sr. Conde de Saldaña y de su hermano el Sr. D. Manuel de Toledo y SalmSalm. (1780).

Archivo-Carpeta 9, 8

124. Bosquexos, descripciones y apuntamientos sobre Botánica (1791-1797).

Archivo-Carpeta 2, 4

125. Delineatio Plantarum.

Archivo-Carpeta 2, 19.

126. [Descripción de plantas]

Archivo-Carpeta 9, 7

127. Descriptiones Plantarum, Laureire Class. 16. (1797).

Archivo-Carpeta 2, 9.

Manuscrito original de una «disertación» leída por Cavanilles en la Real Academia Médica de Madrid.

128. [Diario de las excursiones del viaje a Valencia, con notas, estadísticas, apuntes e informes, todos originales, para la redaccion de sus Observaciones].

2 vols. Archivo-Carpeta 7, 1-2.

Contiene:

- El volumen primero, desde el 20 de marzo de 1792 hasta el 17 de agosto de 1793.

- El volumen segundo, desde el 16 de abril de 1793 hasta el 3 de octubre del mismo año.

129. Hortus Regius Matritensis.

Archivo-Carpeta 9, 2.

Es otro ejemplar manuscrito de 116. 
130. Icones Plantarum.

2 vols. Archivo-Carpeta 7, 5.

Contiene los dibujos originales de Cavanilles para las Icones et descriptiones plantarum, con arreglo a los cuales grabaron sus 600 láminas Alejandro Blanco, José de Fonseca, Miguel Gamborino, Tomás y Vicente López Enguídanos y François Nöel Sellier.

131. Ligeras observaciones sobre los insultos que Mr. Masson de Morvilliers hace a la Nación Española en su artículo "Espagne» de cierta entrega de la Enciclopedia del Diccionario Geográphico.

2 cuadernos. Archivo-Carpeta 2, 1.

Contiene:

- El primer cuaderno, el manuscrito original castellano de las Observations... sur l'article Espagne, diferente de la traducción de Mariano Ribera, que fue impresa en 1784 .

- El segundo cuaderno, una copia del mismo, con notas autógrafas de Cavanilles.

132. [Lista de las piedras pulimentadas y de colecciones efectuadas en el Reino de Valencia]. Archivo-Carpeta 7, 3.

133. [Notas cientificas en relación con la polémica sobre el cultivo de arroces] Archivo-Carpeta 8, 1.

134. [Observaciones de Agricultura Botánica]. Archivo-Carpeta 2, 12.

135. [Observaciones sobre aumento y mejoras del Real Jardín Botánico]. Archivo-Carpeta 2, 15.

136. Observaciones sobre el Reyno de Valencia. 3 vols. Archivo-Carpeta 7.

Contiene:

- Los dos primeros volúmenes, el texto manuscrito original de las Observaciones.

- El tercero, los dibujos originales de Cavanilles para esta obra, con arreglo a los cuales hizo sus grabados Tomás López Enguídanos.

137. Reflexiones para mejorar la enseñanza de las Ciencias Naturales.

Archivo-Carpeta 2, 13.

Copia manuscrita del original de Cavanilles.

\section{Biblioteca Nacional, Madrid}

138. Compendio de la Geografía de España y Portugal, precedido de algunas nociones preliminares y esplicación general de Europa, puesto por preguntas y respuestas para la diversión del Excmo Sr. Conde de Saldaña y su hermano D. Manuel de Toledo (s.a.). $4 .^{\circ}, 63+6+25 \mathrm{~h}$.

Ms. 10776.

139. Apuntamientos lógicos, o compendio del arte lógico-crítco, para el uso del Excmo. Sr. Conde de Saldaña y su hermano el Sr. D. Manuel de Toledo, año 1780.

$4 .^{\circ}, 81 \mathrm{~h}$.

Ms. 10770.

\section{Diputación de Victoria. Fondo "Prestamero»}

140. Informe a la Real sociedad Vascongada, censurando el proyecto de Flora vascongada de D. José de Goyenechea (Madrid, 28 de marzo de 1804).

4. ${ }^{\circ}, 2 \mathrm{~h}$. 


\section{LÁMINAS CALCOGRÁFICAS}

Calcografía Nacional

141. Monadelphia classis dissertationes decem. 104 láminas calcográficas. Catálogo General (167), núms. 1.211-1.314.

Parte de las 296 láminas correspondientes a los grabados de las Dissertationes. Todos son dibujos de Cavanilles, grabados por François Nöel Sellier.

142. Icones et descriptiones plantarum quae aut sponte in Hispania crescunt, aut in Hortis hospitantur.

172 láminas calcográficas.

Catálogo General (167), núms. 1.315-1.486.

Parte de las 600 láminas correspondientes a los grabados de las Icones. Todos los dibujos son de Cavanilles, grabados por Alejandro Blanco, José de Fonseca, Miguel Gamborino, Tomás y Vicente López Enguídanos, y François Nöel Sellier.

143. Hortus Regius Matritensis.

45 láminas calcográficas, 24 × $18 \mathrm{~cm}$.

Catálogo General (167), núms. 1.487-1.531.

Parte de las láminas que se llegaron a grabar, por un grupo de artistas dirigido por Tomás López Enguídanos, a partir de los dibujos del mismo Cavanilles, José Guío y Antonio Delgado Meneses (115) correspondientes a esta obra que Cavanilles dejó inacabada.

144. Observaciones sobre la Historia Natural, geografía, agricultura, población y frutos del Reyno de Valencia.

27 láminas calcográficas.

Catálogo General (167), núms. 3.203-3229.

Parte de las láminas correspondientes a las 53 estampas de la obra, grabadas por Tomas López Enguídanos, conforme a los dibujos de Cavanilles, con la única excepción de las letras del "Mapa del Reyno de Valencia», debidas a José Asensio.

\section{'ESTUDIOS SOBRE CAVANILLES Y SU OBRA}

145. Aguilar Piñal, F.

Bibliografía de autores españoles del siglo XVIII , 7 vols., Madrid, C.S.I.C., 1981-1993. - A. J. Cavanilles: vol. 2, p. 345-351.

146. ALEGRE NÚÑEZ, L.

Catálogo de la Calcografía Nacional, Madrid, Real Academia de Bellas Artes de San Fernando, 1968.

- A. J. Cavanilles: núms. 645, 1935, 1941, 1951, 1954, 1977, 1981, 2031, 2032, 2035, 2037, 2040, 2043, 2120-2147, 2150, 2371, 2388, 2405, 2425-2427, 28473167.

147. Almirante, J.

Bibliografía militar en España, Madrid, M. Tello, 1876.

- A. J. Cavanilles: vol. 1, p. 142.

148. Álvarez López, E.

Cavanilles. Ensayo biografíco-crítico, Anales del Jardín Botánico de Madrid, 6 (1946), 1-64. 
149. Álvarez LÓPEz, E.

Noticia acerca de las plantas estudiadas por Cavanilles y en particular las recolectadas por Luis Née, Revista de Indias, 7 (1946), 502-540 + 7 láms.

150. Álvarez López, E.

Lamarck, Cavanilles y Condillac, Boletín de la Real Sociedad Española de Historia Natural, vol. extra. (1949), 77-87.

151. ANTÓN RAMÍrez, B.

Diccionario de Bibliografía agronómica y de toda clase de escritos relacionados con la Agricultura, Madrid, M. Rivadeneyra, 1865.

- A. J. Cavanilles: p. 305, 504, 548, 578, 607, 632, 874.

152. Archivo del Ilustre Botánico D. Antonio Joseph Cavanilles del que son poseedores los Sres. hermanos Valdes Cavanilles y sus primos, los Sres. hermanos Vigil Cavanilles, Madrid, Nuevas Gráficas, 1946.

153. ASCHERSON, P.F.A.; GRAESNER, K.O.R.P.P.

Synopsis der mitteleuropaischen Flora, 2 vols. Leipzig, W. Engelmann \& Gebr. Borntraeger, 1896-1939.

- A. J. Cavanilles: vol. 2 (1), p. 272.

154. Author Catalogue of the Royal Botanic Gardens Library, Kew, England, 5 vols., Boston, G. K. Hall, 1974.

- A. J. Cavanilles: vol. 1, p. 498.

155. BACKER, C. A.

Verklarend woordenbock der wetenschappelijke namen van de in Nederland en Nederlandsch-Indiè in het wild groeiende en in tuinen en parken gekweekte varens en hoogere planten, Groningen, Noordhoff, 1936.

- A. J. Cavanilles: p. 110.

156. Balguerías Quesada, E.

Una conferencia del Doctor Cavanilles en la Academia de Ciencias de París, Anales del Jardín Botánico de Madrid, 6 (1946), 82-84.

157. Balguerías Quesada, E.

Una carta de D. Mariano Lagasca, Anales del Instituto Botánico Cavanilles, 10 (1951), 119-127.

158. BARNHART, J. H.

Biographical notes upon Botanists, 3 vols., Boston, G. K. Hall, 1965.

- A. J. Cavanilles: vol. 1, p. 327.

159. BARONA VILAR, J.L.

Medicina i història de la ciència en l'obra d'Antoni Josep Cavanilles, Ullal, 7-8 (1985), 47-54.

160. Barona Vilar, J.L.; Micó Navarro, J.A.

Cavanilles y los problemas sanitarios de la Ilustración, Saitabi, 42 (1992), 117-133.

161. BELtrán, F.

Segundo centenario del nacimiento del inmortal botánico José Antonio Cavanilles, Anales del Centro de Cultura Valenciana, 7 (1946), 121-127.

162. Beut y BelENGUeR, E.

Dos excursionistes valencians. Antoni Josep Cavanilles i Antoni Ponz, Valencia, s.i., 1951.

163. BosSERT, TH. W., dir.

Biographical Dictionary of Botanists represented in the Hunt Institute Portrait Collection, Boston, G. K. Hall, 1972.

- A. J. Cavanilles: p. 69

164. Calduch Almela, M.

Castelló i les seues terres en la passada commemoració centenaria de Cavanilles, Boletín de la Sociedad Castellonense de Cultura, 39 (1963), 318-328. 


\section{JOSE M. LOPEZ PIÑERO Y MARIA LUZ LOPEZ TERRADA}

165. CARLES, $\mathrm{C}$.

Cavanilles, Antonio José. En:Diccionario Biográfico de la Ciencia Moderna en Espa$\tilde{n} a$, dir. por J. M. López Piñero, T. F. Glick, V. Navarro y E. Portela, Barcelona, Península, 1983, vol. 1, p. 199-202.

166. [Cavanilles], Botanical Magazine, 2/4 (1788), 186.

167. Catálogo general de la Calcografía Nacional, Madrid, Real Academia de Bellas Artes de San Fernando, 1987.

- A. J. Cavanilles: núms. 1.211-1.531, 3.203-3.229.

168. Catalogue of Scientific Papers, 1800-1900, 19 vols., London, Royal Society, 1867-1925. - A. J. Cavanilles: vol. 1, p. 845-846.

169. Christensen, C.

Index filicum sive enumeratio omnium generum Specierum que filicum et hydropteridum ab anno 1753 ad finem anni 1905 descriptorum, Kjopbenhavn, H. Hagerup, 1906. - A. J. Cavanilles: p. 685

170. Colmeiro, M.

La botánica y los botánicos de la Península Hispano-Lusitana. Estudios bibliográficos y biográficos, Madrid, M. Rivadeneyra, 1858.

- A. J. Cavanilles: p. 119-120, 173-176.

171. DaWson, W. R.

The Banks letters. A. Calendar of the manuscript correspondence of Sir Joseph Banks preserved in the British Museum, the British Museum (Natural History) and other collection in Great Britain, London, British Museum, 1958.

- A. J. Cavanilles: p. 206-207.

172. Enciclopedia universal ilustrada europeo-americana, 100 vols., Bancelona, Espasa, 1905-55. - A. J. Cavanilles: vol. 12, p. 691-692.

173. FERNÁNDEZ RUIZ, C.

El abate don Antonio Joseph Cavanilles, ilustre botánico español de los siglos XVIII y XIX, Medicamenta, 29 (1962), 31-32.

174. FonT QUER, $P$.

En recerca de los Sideritis chamoedryfolia i S. leucanta de Cavanilles, por'González Fragoso, Boletín de la Sociedad Española de Historia Natural, 20 (1920), 201.

175. FONT QUER, P. Sertulum Cavanillesianum enneanthum, Anales del Jardín Botánico de Madrid, 6 (1945), 487-495.

176. GallaCh, J.

Antonio José Cavanilles, La Voz Valenciana, 12 de octubre de 1929, p. 1.

177. García, J. G.

Bicentenario de Cavanilles, Anuario da Sociedade Broteriana, 12 (1946), 9-52.

178. Garganta, M. DE; Camarasa, J. M.

J. M. Cabanilles i Palop, Antoni Josep. En:Gran Enciclopèdia Catalana, Barcelona, Edicions 62, 1969-1980, vol. 4, p. 50.

179. GoNZÁlEZ HERRERo, P.

El bicentenario de Cavanilles, Boletín de la Real Sociedad Española de Historia Natural, 44 (1946) 475-489; 45 (1947), 125-149, 289-305.

180. Gran Enciclopèdia Valenciana, 10 vols., València, Difusora de Cultura Valenciana, 1990-91. - A. J. Cavanilles: vol. 3, p. 24.

181. Gran Larousse Català, 10 vols., Barcelona, Edicions, 62, 1990-93.

- A. J. Cavanilles: vol. 3, p. 1.029.

182. Guillén Marco, V.

La botánica en Valencia después de Linneo. En: Linneo en España. Homenaje a Linneo en su segundo centenario 1707-1907, Zaragoza, M. Escar, 1907, p. 413-428. 
183. Gutiérrez COLOMER, J.

Aspectos poco conocidos de la vida de un botánico español. Antonio José Cavanilles, Anales de la Real Academia de Farmacia, 13 (1947), 47-64.

184. HERNÁNDEZ MOREJÓN, A.

Historia Biobibliográfica de la Medicina Española, 7 vols., Madrid, Imprenta de la Viuda de Jordán e Hijos, 1842-52.

- A. J. Cavanilles: vol. 7, p. 393-397.

185. JACKSON, B. D.

Guide to the Literature of Botany, London, Index Society Publications, 1881.

- A. J. Cavanilles, p. 132 y 139.

186. KeEFE, A. M.

Our debt to the clerical botanists, Biologist, 48 (1966), 45-61.

- A. J. Cavanilles: p. 54.

187. KoENIG; SIMS.

Death of Cavanilles, Annals of Botany, 1/2 (1805), 402-404.

188. LA GASCA, M.

Noticias de la vida literaria de D. Antonio Josef Cavanilles, Variedades de Ciencias, Literatura y Artes, 3 (1804), 65-75, 148-158.

189. LANGMAN, I. K.

A Selected Guide to the Literature on the Flowering Plants of Mexico, Philadelphia, University of Pennsylvania Press, 1964.

- A. J. Cavanilles, p. 194-195.

190. Lasègue, A.

Musée botanique de M. Benjamin Delessert, notices sur les collections de plantes et la bibliographie qui la composent, contenant en outre des documents sur les principaux herbiers d'Europe et l'exposé des voyages entrepis dans l'interet de la botanique, París, Fortin, Masson et Cie., 1845.

- A. J. Cavanilles, p. 564.

191. López PiÑERo, J. M.

[La polémica de la ciencia española durante el siglo XVIII], Investigación y Ciencia, 93 (1984), 6-7.

192. LOPEZ PIÑERO, J.M.

[Antonio José Cavanilles]. En: Historia de la Medicina Valenciana, dir. por J. M. López Piñero, Valencia, Vicent García Eds., 1991, vol II, p. 114-120.

193. LÓPEZ PIÑERO, J.M.

Cavanilles, Antonio José. En: Enciclopedia de Historia de España, dir. por M. Artola, Madrid, Alianza Editorial, vol. 4, 1991, p. 211.

194. LÓPEZ TERRADA, M. L.

La literatura científica en la Valencia de la llustración (1700-1808), Valencia, I.V.E.I., 1987.

- A. J. Cavanilles, p.57-65.

195. Losa España, T.

Algunos comentarios sobre la obra de A. J. Cavanilles: "Observaciones sobre la Historia Natural, la Geografía y la Agricultura del reino de Valencia». Discurso inaugural del año academico 1952-53, Barcelona, Universidad de Barcelona, 1952.

196. MAFFEI, E.; RÚA FIgUEROA, R.

Apuntes para una biblioteca española de libros, folletos y artículos, impresos y manuscritos, relativos al conocimiento y explotacion de las riquezas minerales y a las ciencias auxiliares, 2 vols., Madrid, Imp. J. M. Lapuente, 1871-1872.

- A. J. Cavanilles: vol. 1, p. 144-145. 


\section{JOSE M. LOPEZ PIÑERO Y MARIA LUZ LOPEZ TERRADA}

197. Mansanet y Mansanet, J.; Peris Gisbert, J.

Cavanilles Palop, Antonio José. En:Gran Enciclopedia de la Región Valenciana, Valencia, Difusora de Cultura Valenciana, 1973-77, vol. 3, p. 133:

198. MaTeu Bellés, J. F.

Teorías geomorfológicas europeas en las «Observaciones» de Cavanilles. En: Actas del I Congreso de la Sociedad Española de Historia de las Ciencias, Madrid, Diputación Provincial, 1980, p. 267-287.

199. MATEu TORTOSA, E.

Botánica y agronomía en la España de finales del siglo XVIII: A. Jose Cavanilles y la agricultura valenciana, Valencia, tesis de licenciatura, 1980.

200. Mateu Tortosa, E.

Arroz y paludismo, Valencia, I.V.E.I., 1987.

- A.J. Cavanilles: p.125-132.

201. Meliá Tena, C.

L'economia del Regne de Valencia segons Cavanilles, Valencia, L'Estel, 1978.

202. MENÉNDEZ AMOR, J.

Segundo centenario del nacimiento del ilustre botánico español, don Antonio José Cavanilles y Palop, Boletín de la Real Sociedad Española de Historia Natural, 44 (1946), 249-252.

203. MERRIL, E. D.; WALKER, E. H.

A Bibliography of Eastern Asiatic Botany, Jamaica Plain, Mass., The Arnold Arboretum of Harvard University, 1938.

- A. J. Cavanilles, p. 71.

204. Micó NaVARro, J.A.; Barona VilaR, J.L.

La Higiene y sanidad de las comarcas castellonenses en el siglo XVIII a través de las "Observaciones..." de Cabanilles, Estudis Catellonencs, 5 (1993), 467-482.

205. MONGE MartíneZ, F.

La historia natural y moral en la obra de A.J. Cavanilles, 1745-1804, Revista de Indias, 52 (1991), 693-722.

206. NISSEN, C.

Die Botanische Buchillustration. Ihre Geschichte und Bibliographie, 2. ${ }^{\mathrm{a}}$ ed., 2 vols., Stuttgart, A. Hiersemann, 1966.

- A. J. Cavanilles: vol. 1, p. 157-158; vol. 2, p. 33.

207. Palau Dulcet, A.

Manual del librero hispano-americano, 28 vols., Barcelona [-Oxford], A. Palau [y The Dolphin Books], 1948-1977.

- A. J. Cavanilles: vol. 3, p. 347

208. Pascual, $R$.

Cavanilles y el tratamiento de la rabia. En:Actas del III Congreso Nacional de Historia de la Medicina, Valencia, Sociedad Española de Historia de la Medicina, 1969, vol. II, 287-294.

209. PASTOR FUSTER, J.

Biblioteca valenciana de los escritores que florecieron hasta nuestros días. Con adiciones y enmiendas a la de D. José Ximeno, 2 vols., Valencia, Imprenta y Librería de José Ximeno, 1827-30.

- A. J. Cavanilles: vol. 2, p. 256-260.

210. Pelayo, F.

Las actividades botánicas del naturalista valenciano A.J. Cavanilles, Madrid, Real Jardín Botánico, 1990. 


\section{BIBLIOGRAFIA DE ANTONIO JOSE CAVANILLES (1745-1804)}

211. Pelayo, F.; Garilleti, $R$.

La formación y las actividades botánicas de A.J. Cavanilles, Asclepio, 44 / 2 (1992), 129-154.

212. Peset Reig, M.; Peset Reig, J. L.

Muerte en España (Política y sociedad entre la peste y el cólera), Madrid, Seminarios y Ediciones, 1972.

- A. J. Cavanilles: p. 44-68

213. Peset Reig, M.; Peset Reig, J. L.

Cultivos de arroz y paludismo en la Valencia del siglo XVIII, Hispania, 32 (1972), 277-376.

214. Petit-Thovars, D

Cavanilles, Antoine Joseph. En: Biographie universelle ancienne et moderne... Nouvelle edition publiée sous la direction de M. Michaud, vol. 7, París, Desplaces, 1854 p. 267-269.

215. Pires de Lima, A.

Tres cartas inéditas de Brotero a Cavanilles. Anuario da Sociedade Broteriana, 12 (1946), 53-92.

216. Pizcueta Donday, J.

Elogio histórico de Don Antonio José Cavanilles, Valencia, Benito Monfort, 1830

2. ${ }^{a}$ ed., Madrid, Imp. del Asilo de Huérfanos del Sagrado Corazón, 1906.

217. PRITZEL, G. A.

Thesaurus litteraturae botanicae, Leipzig, F.A. Brockhaus, 1851.

- A. J. Cavanilles: p. 1762-1769.

218. QUENSTEDT, W.; LAMBRECHT, A. K

Fossilium catalogus, 's-Gravenhage, 1938.

- A. J. Cavanilles: p. 77.

219. ReYes PRÓSPER, E.

Dos noticias históricas del inmortal botánico y sacerdote hispanovalentino Don Antonio José Cavanilles y Ceuti y Don Mariano Lagasca. Con anotaciones y los estudios bio-bibliográficos de Cavanilles y Ceuti y de La Gasca, Madrid, Artes Gráficas Mateu, 1917.

La Noticia histórica del Señor Don Antonio José Cavanilles, de Antonio Cavanilles Ceutí, había sido leída por su autor en la Real Academia de la Historia en 1841. La de La Gasca había sido publicada en 1804 (183).

220. RIVAS GODAY, S.

Dos plantas cavanillesianas (y consideraciones sobre la vegetación de las comarcas en donde habitan), Anales del Jardín Botánico de Madrid, 6 (1945); 397-420 + láms.

221. Rivas Goday, S.

Cavanilles, precursor de la geografía botánica. Anales de la Academia de Farmacia, 40 (1974), 403-419.

222. ROBRES, $R$.

Cavanilles, Antonio José. En: Diccionario de Historia eclesiástica de España, dir. por Q.A. Vaquero, T.M. Martínez y J.V. Gatell, vol. 1, Madrid, C.S.I.C., 1972, p. 391-392.

223. Ron ÁlVAREZ, M.E.

Cavanilles, Antonio José. En: Gran Enciclopedia Rialp, vol. 5, Madrid, 1979, p. 411-412.

224. Rosselló Verger, V.M.; LóPEz Piñero, J.M.; Costa TAléns, M. et al. Cavanilles, Naturalista de la Il.lustració.1745-1803, Valencia, Universidad de Valencia, 1983.

225. Rosselló Verger, V.M.; LóPez Piñero, J.M.; Costa TAlÉNS, M. et al. Cavanilles, Naturalista de la Ilustración, 1745-1803, Madrid, Real Jardín Botánico, 1983. 


\section{JOSE M. LOPEZ PIÑERO Y MARIA LUZ LOPEZ TERRADA}

226. ROTHMALER, W.

["Elenchus plantarum horti regii botanici matritensis», de A. J. Cavanilles], Repertorium specierum novarum regni vegetalis. Centralblatt für Sammlung und Veröffentlichung vor Einzeldiagnosen neuer Pflanzen, 49 (1940), 51-53.

227. Salvá y MaLlÉN, P.

Catálogo de la biblioteca Salvá, 2 vols., Valencia, Imprenta de Ferrer de Orga, 1872. - A. J. Cavanilles: vol. 2, p. 273, 327, 397, 454

228. SCHRADER, H. A.

[A. J. Cavanilles], Neues Journal für die Botanik, 1 (1807), 150-162.

229. SEMPERE GUARINOS, J.

Ensayo de una biblioteca española de los mejores escritores del Reynado de Carlos III, 6 vols., Madrid, Imprenta Real, 1785.

- A. J. Cavanilles: vol. 2, p. 166-172.

230. Stafleu, F. A.

[A. J. Cavanilles], Taxon. International Association for Plant Taxonomy, 21 (1972), 138.

231. Stafleu, F. A.; Cowan, R. S.

Taxonomic Literature. A Selective Guide to Botanical Publications and Collections with Dates, Commentaries and Types, 2. ${ }^{a}$ ed., vol. I: A-G. Utrecht, Bohn, Scheltema \& Holkema, 1976.

- A. J. Cavanilles: p. 471-474.

232. Terrada FERrandis, M. L.

[Antonio José Cavanilles], En: La anatomia microscopica en España (siglos XVIIXVIII), Salamanca, Seminario de Historia de la Medicina, 1969, p. 59-65.

233. VALDERAS, J.M.

Relectura de las Dissertaciones de Cavanilles, Collectanea Botánica, 20 (1991), 183-238.

234. Van KaAthoven, C. W. H.

Portraits de médecins, et gravures ayant rapport à l'histoire de la médecine, offrant des types et des charges des médecins et de chirurgiens de tous les temps.

Colección de 12 vols. reunida por Van Kaathoven y vendida en 1879 a la National Library of Medicine, de Bethesda, donde se conserva. En ella figura un retrato de "Antonius Joseph Cavanilles», grabado por F. Neyer en Erfurt, 1805, que fue publicado en el artículo de H. A. Schrader antes citado (225).

235. Vegas Fabián, G.

El eminente Cavanilles (1745-1805), Madrid, s.i., 1946.

236. VERNET, J.

Cavanilles, Antonio José, Dictionary of Scientific Biography, dir. por C. C. Gillispie, vol. 3, New York, Charles Scribner's Sons, 1971, p. 154-155.

237. ZANDER, A.

Handwörterbuch der Pflanzennamen. Neu bearbeitete und erweiterte 10. Auflage von Dr.h.c. Fritz Encke und Dr. Gunther Buchheim unter Mitarbeit von Dr. Siegmund Seybeld, Stuttgart, Enke, 1972.

- A. J. Cavanilles: p. 644.

238. ZARAGOZA RUBIRA, J. R.

Un estudio médico sobre el cultivo del arroz en la España del siglo XVIII. (Sobre una memoria de Antonio José Cavanilles), Medicina Española, 50 (1963), p. 318327.

239. ZuRriaga Agustí, F.

El paisatge agrari del Camp de Túria en l'obra d'Antoni Josep Cavanilles (17451808), Lauro. Quaderns d'història i societat, 6 (1992), 175-182. 